\title{
Star formation in AGN hosts in GOODS-N ${ }^{\star}$
}

\author{
L. Shao ${ }^{1}$, D. Lutz ${ }^{1}$, R. Nordon ${ }^{1}$, R. Maiolino ${ }^{2}$, D. M. Alexander ${ }^{3}$, B. Altieri ${ }^{4}$, P. Andreani ${ }^{5,6}$, H. Aussel ${ }^{7}$, F. E. Bauer ${ }^{8}$, \\ S. Berta ${ }^{1}$, A. Bongiovanni ${ }^{9}, 10$, W. N. Brandt ${ }^{11}$, M. Brusa ${ }^{1}$, A. Cava ${ }^{9,10}$, J. Cepa ${ }^{9,10}$, A. Cimatti ${ }^{12}$, E. Daddi ${ }^{7}$, \\ H. Dominguez-Sanchez ${ }^{12}$, D. Elbaz ${ }^{7}$, N. M. Förster Schreiber ${ }^{1}$, N. Geis ${ }^{1}$, R. Genzel ${ }^{1}$, A. Grazian ${ }^{2}$, C. Gruppioni ${ }^{12}$, \\ G. Magdis ${ }^{7}$, B. Magnelli ${ }^{1}$, V. Mainieri ${ }^{5}$, A. M. Pérez García, ${ }^{9},{ }^{10}$, A. Poglitsch ${ }^{1}$, P. Popesso ${ }^{1}$, F. Pozzi ${ }^{12}$, L. Riguccini ${ }^{7}$, \\ G. Rodighiero ${ }^{13}$, E. Rovilos ${ }^{1}$, A. Saintonge ${ }^{1}$, M. Salvato ${ }^{14}$, M. Sanchez Portal ${ }^{4}$, P. Santini ${ }^{2}$, E. Sturm ${ }^{1}$, L. J. Tacconi ${ }^{1}$, \\ I. Valtchanov ${ }^{4}$, M. Wetzstein ${ }^{1}$, and E. Wieprecht ${ }^{1}$
}

(Affiliations are available in the online edition)

Received 31 March 2010 / Accepted 13 May 2010

\begin{abstract}
Sensitive Herschel far-infrared observations can break degeneracies that were inherent to previous studies of star formation in high- $z$ AGN hosts. Combining PACS 100 and $160 \mu \mathrm{m}$ observations of the GOODS-N field with 2 Ms Chandra data, we detect $\sim 20 \%$ of X-ray AGN individually at $>3 \sigma$. The host far-infrared luminosity of AGN with $L_{2-10 \mathrm{keV}} \approx 10^{43} \mathrm{erg} \mathrm{s}^{-1}$ increases with redshift by an order of magnitude from $z=0$ to $z \sim 1$. In contrast, there is little dependence of far-infrared luminosity on AGN luminosity, for $L_{2-10 \mathrm{keV}} \lesssim 10^{44} \mathrm{erg} \mathrm{s}^{-1} \mathrm{AGN}$ at $z \gtrsim 1$. We do not find a dependence of far-infrared luminosity on X-ray obscuring column, for our sample which is dominated by $L_{2-10 \mathrm{keV}}<10^{44} \mathrm{erg} \mathrm{s}^{-1}$ AGN. In conjunction with properties of local and luminous high- $z$ AGN, we interpret these results as reflecting the interplay between two paths of AGN/host coevolution. A correlation of AGN luminosity and host star formation is traced locally over a wide range of luminosities and also extends to luminous high-z. AGN. This correlation reflects an evolutionary connection, likely via merging. For lower AGN luminosities, star formation is similar to that in non-active massive galaxies and shows little dependence on AGN luminosity. The level of this secular, non-merger driven star formation increasingly dominates over the correlation at increasing redshift.
\end{abstract}

Key words. galaxies: active - infrared: galaxies

\section{Introduction}

Measuring the star formation rate of the host galaxy is important for studying the co-evolution of active galactic nuclei (AGN) and their hosts. It is often difficult because the AGN can outshine the host at many wavelengths. However, the rest frame far-infrared/submm emission appears dominated by the host for AGN with $v L_{v}(60 \mu \mathrm{m}) \approx 0.1 \ldots 0.2 L_{\mathrm{Bol}, \mathrm{AGN}}$ and higher (e.g. Netzer et al. 2007; and introduction to Lutz et al. 2010; see also Wang et al. 2008 for luminous high-z QSOs) and has been used as a host star formation rate diagnostic of high- $z$ AGN (e.g. Serjeant \& Hatziminaoglou 2009; Mullaney et al. 2010; and Lutz et al. 2010). Herschel can detect much lower star formation rates than previous far-infrared and submm studies. It further improves such work by measuring the rest frame far-infrared SED peak without extrapolation from longer wavelengths. We here present a first Herschel study of rest frame far-infrared emission and host star formation in X-ray selected AGN in the GOODS-N field. Throughout the paper, we adopt an $\Omega_{\mathrm{m}}=0.3, \Omega_{\Lambda}=0.7$ and $H_{0}=70 \mathrm{~km} \mathrm{~s}^{-1} \mathrm{Mpc}^{-1}$ cosmology.

\section{Data}

We use the v2.2 100 and $160 \mu \mathrm{m}$ maps of the GOODS-N field obtained with PACS (Poglitsch et al. 2010) on board Herschel

* Herschel is an ESA space observatory with science instruments provided by European-led Principal Investigator consortia and with important participation from NASA.
(Pilbratt et al. 2010) as a science demonstration project for the PACS evolutionary probe (PEP) guaranteed time survey. We use the PACS catalog extracted with IRAC/MIPS $24 \mu \mathrm{m}$ based position priors by B. Magnelli, to a $3 \sigma$ depth of $\sim 3.0 \mathrm{mJy}$ and $\sim 5.7 \mathrm{mJy}$ at 100 and $160 \mu \mathrm{m}$, respectively. Since there are Chandra sources that are not detected at $24 \mu \mathrm{m}$, we have verified by individual comparison to a blind catalog that we are not missing PACS detections on such sources when using the $24 \mu \mathrm{m}$ prior catalog. For samples of objects undetected by PACS we stack, using the X-ray positions, into the residual maps obtained after subtracting the sources in the prior catalog from the original maps. See also the appendix to Berta et al. (2010) for a description of the data and catalogs.

The CDFN 2 Ms Chandra X-ray catalog of Alexander et al. (2003) provides the basis for our AGN selection. 328 of its 503 X-ray sources lie within the part of the PACS map, which has at least half of the coverage of the central homogeneously covered region; we restrict ourselves to this subset. Reaching $\approx 2.5 \times 10^{-17} \mathrm{erg} \mathrm{cm}^{-2} \mathrm{~s}^{-1}$ in the $0.5-2 \mathrm{keV}$ band, CDFN X-ray detections include a noticeable number of star forming galaxies without significant AGN contributions, in addition to bonafide AGN. Bauer et al. (2004) have outlined for this very sample a combination of criteria to distinguish X-ray AGN from star formation dominated objects, based on X-ray luminosity, X-ray obscuring column or hardness, optical spectroscopic classifications, and X-ray/optical flux ratio. We use an updated version of their classifications for the GOODS-N X-ray sources, which separates the 328 sources with good PACS coverage into 224 X-ray 
Table 1. PACS detections of GOODS-N X-ray sources.

\begin{tabular}{lcccc}
\hline \hline Class & \multirow{2}{*}{ Sources } & \multicolumn{3}{c}{$>3 \sigma$ detections at } \\
& & $100 \mu \mathrm{m}$ & $160 \mu \mathrm{m}$ & 100 or $160 \mu \mathrm{m}$ \\
\hline All & 328 & 76 & 81 & 91 \\
Galaxies & 67 & 37 & 37 & 40 \\
AGN & 224 & 35 & 39 & 46 \\
Other & 37 & 4 & 5 & 5 \\
\hline
\end{tabular}

AGN, 67 X-ray detected star forming galaxies, and 37 other targets (stars or unidentified). We use both spectroscopic (Barger et al. 2008, 57\% of our X-ray AGN) and photometric redshifts (F. Bauer, mostly based on Barger et al. 2003). X-ray spectral fitting (F. Bauer et al., in prep.) provides intrinsic X-ray luminosities $L_{2-10 \mathrm{keV}}$ and obscuring columns $N_{\mathrm{H}}$ for the X-ray sources. The fits use absorbed powerlaws with a fixed Galactic column of $1.6 \times 10^{20} \mathrm{~cm}^{-2}$ and a variable obscuring column at the redshift of the source. The photon index is allowed to vary for sources above 150 net counts in the $0.5-8 \mathrm{keV}$ band, and is fixed to 1.85 below.

\section{Results}

The far-infrared detection rate of the 328 X-ray sources above the $3 \sigma$ level in at least one of the two PACS bands is $28 \%$ (Table 1). For the three subgroups, it is 60\% (galaxies), $21 \%$ (AGN) and 14\% (other). The large detection rate of "galaxies" is consistent with their X-ray emission arising from star formation related processes. A detailed discussion will be provided elsewhere. In the following, we focus on the 224 X-ray AGN only.

We use both the individual detections and stacks of nondetections derived via the stacking library of Bethermin et al. (2010). The 178 AGN that are individually undetected in both PACS bands are statistically well detected in the stacks at $0.79 \pm$ $0.08 \mathrm{mJy}(100 \mu \mathrm{m})$ and $1.46 \pm 0.16 \mathrm{mJy}(160 \mu \mathrm{m})$. These detection errors are derived from multiple stacks at random positions over the same coverage region of the PACS residual maps, and using the number of targets as the sample of interest.

To derive far-infrared (FIR) luminosities with minimal assumptions about SED shapewe compute the rest frame $60 \mu \mathrm{m}$ luminosity $v L_{v}(60 \mu \mathrm{m})$ using the detection wavelength closer to rest $60 \mu \mathrm{m}$ or log-linearly interpolating for detections in both bands at $0.67<z<1.67$. We treat stacked $100 \mu \mathrm{m}$ and $160 \mu \mathrm{m}$ fluxes equivalently to derive the mean rest frame $60 \mu \mathrm{m}$ luminosities of the individually undetected sources. Given the strong $K$-corrections for PACS fluxes over the redshift range of X-ray AGN, this requires restricted redshift ranges for the stacks, we limit to bins with $\Delta z /(1+z)<0.4$ and adopt the median $z$ of the particular sample. Luminosities for the combined sample of detections and nondetections are obtained as averages weighted by the number of sources.

Figure 1 shows the redshift distribution of all X-ray AGN in our sample. As expected, PACS photometry is most efficient in detecting $z \lesssim 2.5$ AGN hosts. We also focus on $z \leq 2.5$ in order to probe far-infrared rest frame $>45 \mu \mathrm{m}$ wavelengths, beyond the mid-infrared that is more easily AGN dominated. Table 2 and Fig. 2 show $60 \mu \mathrm{m}$ luminosities as a function of redshift, separately for the PACS detections, stacks of nondetections and the combined sample. Host $60 \mu \mathrm{m}$ luminosities increase with redshift. An increase is seen in detections, stacked nondetections, and in the averages for the combined sample. This trend cannot be simply due to the increase of FIR detection limit with redshift, which would leave the average luminosities from the combination of detections and nondetections unchanged.

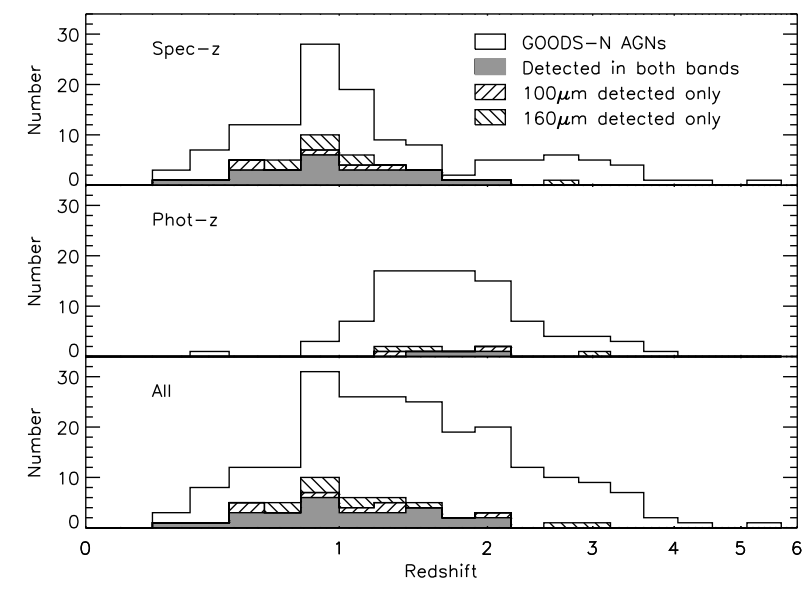

Fig. 1. Redshift distribution of the 224 GOODS-N X-ray AGN in the region with good PACS coverage. 46/224 are individually detected in at least one of the PACS bands.

Table 2. Mean FIR luminosities of different AGN groups.

\begin{tabular}{|c|c|c|c|c|c|}
\hline$z$ & $\begin{array}{l}L_{2-10 \mathrm{keV}} \\
\mathrm{erg} \mathrm{s}^{-1}\end{array}$ & $N_{\text {Det }} / N$ & $\begin{array}{r}\text { Detected } \\
v L_{v}(60\end{array}$ & $\begin{array}{l}\text { Stack } \\
\text { m rest) }\end{array}$ & $\begin{array}{r}\text { All } \\
0^{44} \mathrm{erg} \mathrm{s}^{-1}\end{array}$ \\
\hline $0.2-0.8$ & all & $12 / 35$ & 3.3 & 0.32 & $1.33 \pm 0.31$ \\
\hline $0.8-1.4$ & all & $22 / 83$ & 11.3 & 1.43 & $4.04 \pm 0.62$ \\
\hline $1.4-2.5$ & all & $10 / 76$ & 61.1 & 5.27 & $12.61 \pm 3.12$ \\
\hline $0.2-0.8$ & $<10^{42}$ & $4 / 16$ & 1.9 & 0.09 & $0.55 \pm 0.23$ \\
\hline $0.8-1.4$ & $<10^{42}$ & $1 / 9$ & 6.9 & 1.40 & $2.01 \pm 0.91$ \\
\hline $1.4-2.5$ & $<10^{42}$ & $0 / 1$ & & 3.92 & \\
\hline $0.2-0.8$ & $10^{42-43}$ & $2 / 12$ & 4.4 & 0.60 & $1.23 \pm 0.51$ \\
\hline $0.8-1.4$ & $10^{42-43}$ & $7 / 37$ & 12.3 & 1.56 & $3.60 \pm 0.73$ \\
\hline $1.4-2.5$ & $10^{42-43}$ & $6 / 18$ & 63.4 & 4.35 & $24.04 \pm 8.45$ \\
\hline $0.2-0.8$ & $10^{43-44}$ & $6 / 7$ & 3.8 & 1.91 & $3.54 \pm 0.89$ \\
\hline $0.8-1.4$ & $10^{43-44}$ & $13 / 32$ & 10.5 & 1.61 & $5.22 \pm 1.11$ \\
\hline $1.4-2.5$ & $10^{43-44}$ & $3 / 51$ & 69.3 & 5.30 & $9.06 \pm 2.80$ \\
\hline $0.2-0.8$ & $>10^{44}$ & $0 / 0$ & & & \\
\hline $0.8-1.4$ & $>10^{44}$ & $1 / 5$ & 18.2 & 2.32 & $5.50 \pm 3.56$ \\
\hline $1.4-2.5$ & $>10^{44}$ & $1 / 6$ & 22.2 & 8.58 & $10.85 \pm 4.77$ \\
\hline \multirow[t]{2}{*}{2} & \multirow{2}{*}{$\begin{array}{c}N_{\mathrm{H}} \\
\mathrm{cm}^{-2}\end{array}$} & $N_{\text {Det }} / N$ & \multirow{2}{*}{\multicolumn{3}{|c|}{$\begin{array}{c}\text { Stack } \\
\mu \mathrm{m} \text { rest }), 10^{44} \mathrm{erg} \mathrm{s}^{-1}\end{array}$}} \\
\hline & & & & & \\
\hline $0.8-1.4$ & $<10^{22}$ & $2 / 10$ & 13.7 & 1.12 & $4.15 \pm 1.25$ \\
\hline $0.8-1.4$ & $10^{22-23}$ & $6 / 25$ & 11.6 & 1.47 & $3.89 \pm 0.91$ \\
\hline $0.8-1.4$ & $10^{23-24}$ & $10 / 28$ & 9.6 & 1.76 & $4.57 \pm 0.89$ \\
\hline $0.8-1.4$ & $>10^{24}$ & $0 / 5$ & & 1.67 & $1.67 \pm 0.88$ \\
\hline
\end{tabular}

Notes. Average FIR luminosities are given separately for the individual $>3 \sigma$ detections, the stack of the nondetections, and the numberweighted mean for all sources. Errors for luminosities are standard deviations from bootstrapping and are dominated by the dispersion of the AGN population rather than measurement error.

Mean fluxes for the PACS $>3 \sigma$ detections and stacked mean for the nondetections differ by about an order of magnitude in each of the three redshift bins. Such a large difference must reflect a wide intrinsic distribution of far-infrared luminosities. For the simplifying assumption of a log-normal distribution of farinfrared luminosities, the ratio of mean detections to the stacked mean as well as the $\sim 20 \%$ detection rates can be reproduced with an intrinsic dispersion of about 0.5 dex for the distribution of farinfrared luminosities in each bin. The detailed shape of this distribution is not constrained but must be wide. The mean $160 \mu \mathrm{m}$ flux for all the $1.4<z<2.5 \mathrm{AGN}$ is $3 \mathrm{mJy}$ (in agreement with tentative MIPS values for ECDFS AGN by Papovich et al. 2007). Given the wide distribution of FIR luminosities, the typical (median) flux must be lower, by a factor $\sim 2$ for the log-normal 


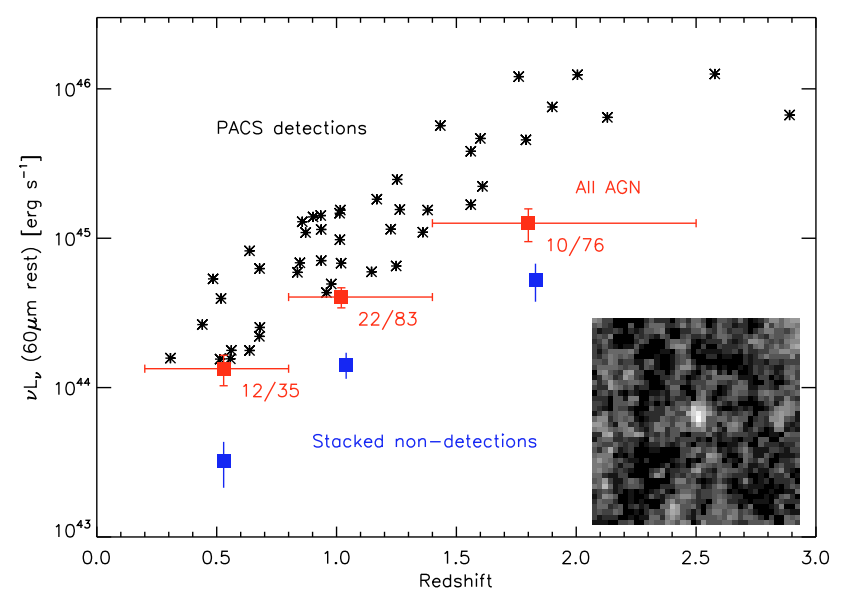

Fig. 2. Far-infrared luminosities of GOODS-N X-ray AGN as a function of redshift. Symbols labelled "All AGN", with the redshift range indicated, average the detections and nondetections. Their uncertainty is derived from bootstrapping into the combined sample. Number of detections and total number of sources in each bin are indicated. The insert shows the stack for the indvidually undetected $0.8<z<1.4$ sources.

distribution. A considerable variety exists not only in far-infrared luminosity but also in mid-to far-infrared SED shape (Fig. 3), from SEDs quite similar to those of star forming galaxies to ones that are clearly AGN dominated over a wide wavelength range.

The increase of host far-infrared emission with redshift in Fig. 2 could still be influenced by the increase of AGN luminosity with redshift that is inherent to this X-ray selected sample. To break possible degeneracies, we have binned our sample in $3 \times 4$ bins defined by redshift $(z=0.2-0.8,0.8-1.4,1.4-2.5)$ and by intrinsic hard X-ray luminosity $\left(L_{2-10 \mathrm{keV}}<10^{42} \mathrm{erg} \mathrm{s}^{-1}\right.$, $\left.10^{42}-10^{43}, 10^{43}-10^{44},>10^{44}\right)$. Table 2 lists FIR luminosities for these bins, for the figures in the following discussion we omit bins with less than 5 objects and correspondingly large errors. Errors on the average FIR luminosity are dominated by the variations in the underlying population rather than by photometric error. For that reason, errors in Table 2 and the figures are standard deviations derived from bootstrap estimates for the respective subsamples.

Figure 4 (left) shows that the increase of host FIR luminosity with redshift is clearly preserved when considering AGN luminosity bins separately. Focusing on the far-infrared luminosities of $L_{2-10 \mathrm{keV}}=10^{42}-10^{43} \mathrm{AGN}$, for which there are more than 10 objects in each redshift bin, there is no overlap in the $99 \%$ confidence intervals of FIR luminosity comparing the $z=0.2-0.8$ and the $z=1.4-2.5$ redshift range $\left(<2.8\right.$ vs. $\left.>6.1 \times 10^{44} \mathrm{erg} \mathrm{s}^{-1}\right)$. These $99 \%$ confidence intervals were directly derived by bootstrapping and thus include nonGaussianity of distributions of individual source properties or of errors. The higher the AGN luminosity bin we consider, the higher is the FIR luminosity at low $z$ but then its increase with redshift less steep. Conversely, when studying FIR luminosity as a function of AGN luminosity (Fig. 4 right) there is an increase with AGN luminosity in the lowest redshift bin that flattens at higher redshift, with no significant trend left at $z>1.4$.

In the luminosity range covered by our sample, we do not find a significant trend of FIR luminosity with X-ray obscuring column (Fig. 5). Such a trend would be expected in merger evolutionary scenarios that are invoking a sequence starburst obscured AGN - unobscured AGN (e.g. Sanders et al. 1988; Hopkins et al. 2006).

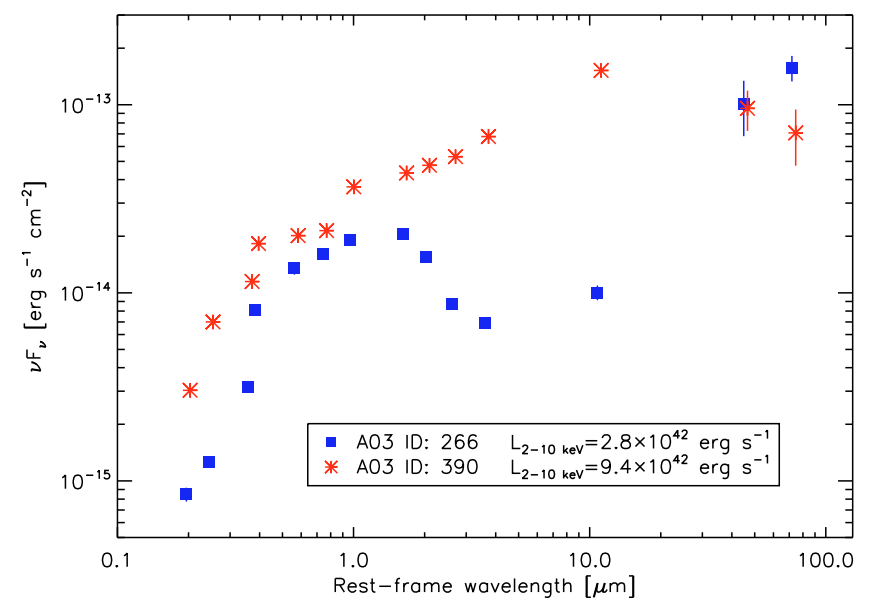

Fig. 3. Examples of $z \sim 1.2 \mathrm{AGN}$ illustrating the range of optical to farinfrared SEDs. ID 390 (Alexander et al. 2003) is dominated by AGN continuum over the optical to mid-infrared, while ID 266 which hosts an high obscuring column AGN is dominated by an optical/NIR stellar bump and a pronounced far-infrared peak.

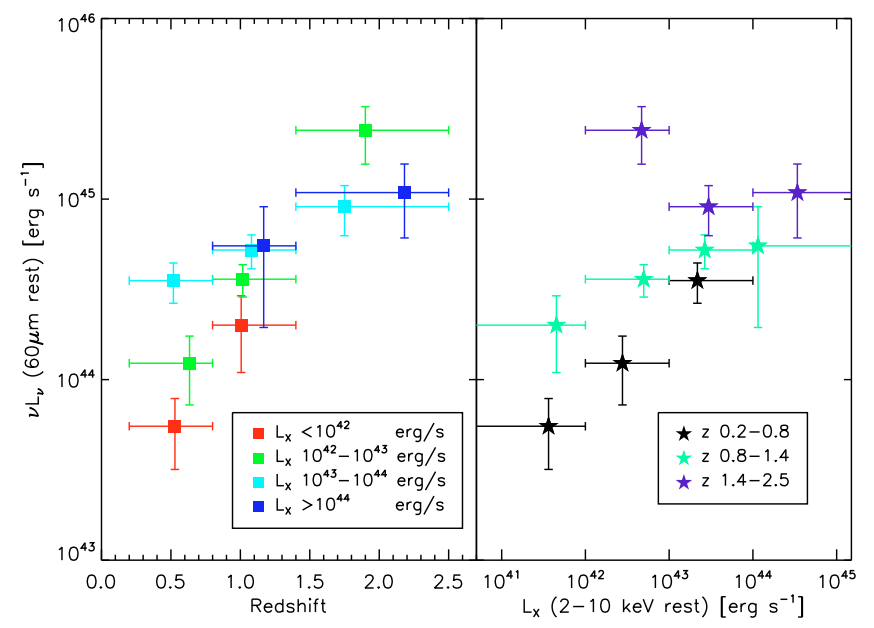

Fig. 4. Left: far-infrared luminosity as a function of redshift, for different bins in intrinsic rest frame $2-10 \mathrm{keV} X$-ray luminosity. Values reflect the mean of detections and nondetections, and errors are based on bootstrapping into the respective sample. Right: FIR luminosity as a function of intrinsic hard X-ray luminosity, for different redshift bins.

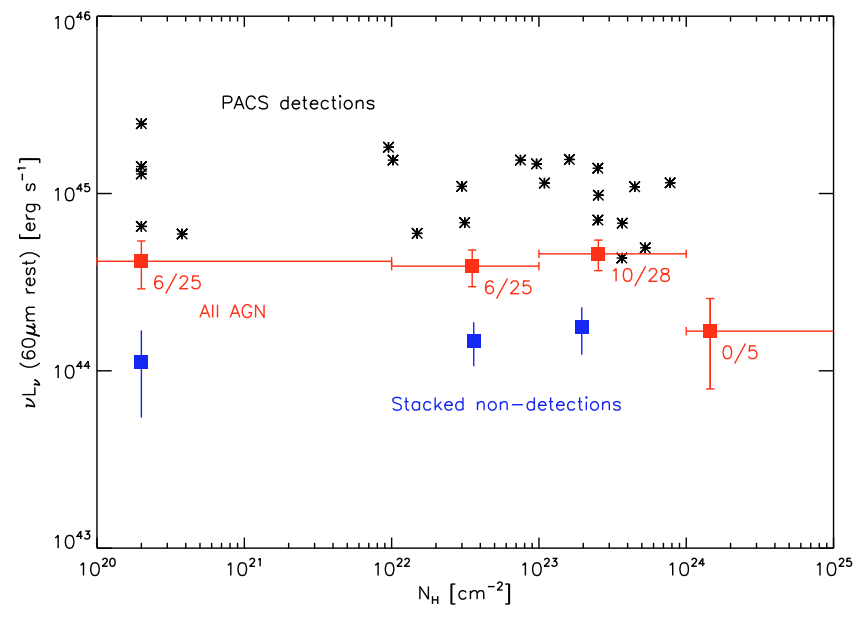

Fig. 5. Far-infrared luminosity as a function of X-ray obscuring column. Sample for redshifts $0.8<z<1$.4. Low obscuring column objects have been placed at $2 \times 10^{20} \mathrm{~cm}^{-2}$. Symbols are as in Fig. 2 . 


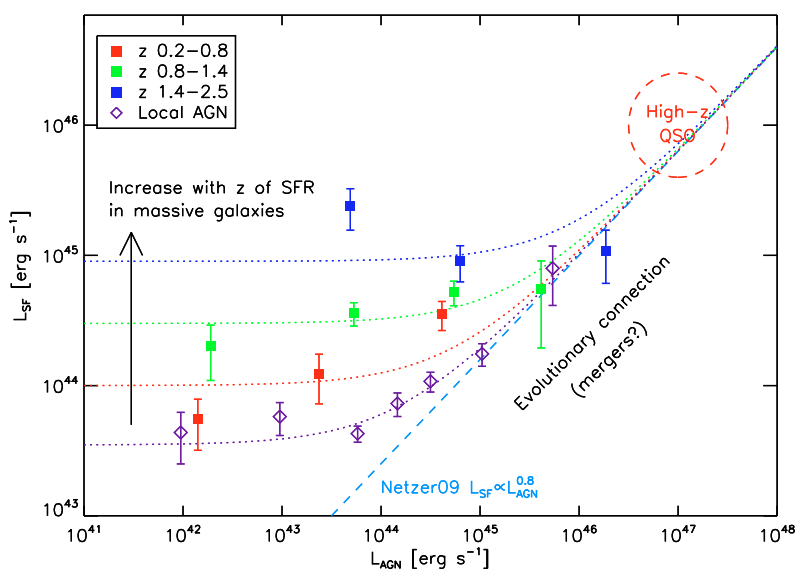

Fig. 6. Star forming (=far-infrared) luminosity vs. AGN luminosity for the GOODS-N AGN and a local reference sample of extremely hard X-ray selected BAT AGN. The dotted colored lines indicate schematically how the observations are explained by the combination of a diagonal "evolutionary connection" trend with a general increase of host star formation with redshift in hosts of moderate luminosity AGN, similar to that for the general galaxy population. The dashed line is the relation implied by Netzer et al. (2009).

\section{Evolution of the relation between AGN luminosity and host star formation}

Due to the excellent sensitivity of Herschel PACS to rest frame far-infrared emission in the hosts of $z=0.2-2.5 \mathrm{X}$-ray selected AGN, we have been able to break the redshift/luminosity degeneracy that affected previous submm-based studies of AGN host star formation (e.g. Lutz et al. 2010). We avoid having to make SED assumptions that were necessary for submm-based studies. Compared to the Spitzer $70 \mu \mathrm{m}$-based study of Mullaney et al. (2010), we are probing the rest frame far-infrared out to $z \gtrsim 1$ where $70 \mu \mathrm{m}$ data already enter the rest frame mid-IR with less favourable contrast between host emission and AGN heated dust. Results of these submm as well as $70 \mu \mathrm{m}$ based studies agree well with our findings within the mentioned constraints. To study evolution over a yet wider redshift range, we supplement in the following our GOODS-N sample with local $(z<0.3)$ Swift BAT $14-150 \mathrm{keV}$ extremely hard X-ray detected AGN (Cusumano 2009). Here, far-infrared information is taken from the IRAS database, in a way consistent with the treatment of the GOODS-N AGN (see also Sect. 3.3 of Lutz et al. 2010).

A variety of studies have established a local correlation between AGN luminosity and star forming luminosity (e.g. Rowan-Robinson 1995; Netzer et al. 2007, 2009), at least in the luminosity regime of bright Seyferts or Quasars. High redshift QSOs appear to extend this relation to yet higher luminosities (e.g. Lutz et al. 2008 and references therein). Figure 6 places our results in that context. Here we have converted from hard $\mathrm{X}$-ray to AGN bolometric luminosity using Eq. (5) of Maiolino et al. (2007) and a ratio 7 between bolometric and $5100 \AA$ luminosity. Local BAT AGN follow the correlation of AGN and star formation luminosity, with the exception of a flattening at low AGN luminosities. Figure 6 shows that high luminosity AGN stay close to the correlation for all redshifts covered by our sample, but the host star formation rates of lower luminosity AGN rise by about an order of magnitude from $z=0$ to $z=1$ and by about 1.5 orders of magnitude from $z=0$ to $z=2$. Such a behavour can be explained by the combination of two paths of AGN growth. On one path, AGN growth and host star formation are tightly coupled by an evolutionary mechanism, likely merging. This will result in the diagonal correlation line in Fig. 6. The other path reflects a secular evolution with no close coupling of AGN luminosity and galaxy-integrated host star formation rate. Connections between AGN and star formation phenomena on smaller spatial scales or different timescales might however be present. Here, star formation levels will be similar to those that are pervasive in massive galaxies at a given redshift. This corresponds to the low-luminosity flattening of the relation in Fig. 6 at a level increasing with redshift. The detailed slope of this flatter part is still poorly constrained at current statistics. Bouché et al. (2010) have parametrized on the basis of a variety of observational studies star formation rates of star forming galaxies as a function of galaxy mass and redshift. Their Eq. (1) corresponds to an increase in SFR from redshift 0 to the centers of our three redshift bins by factors 3.0, 7.4, and 19 respectively, consistent with the location of AGN on the flat "secular" path in Fig. 6. As discussed in Lutz et al. (2010), such a two-path scenario for AGN is also consistent with a variety of other properties of AGN hosts at high $z$.

A limitation to the current study is the small $\sim 11^{\prime} \times 16^{\prime}$ field which restricts the number of $L_{2-10 \mathrm{keV}}>10^{44} \mathrm{erg} \mathrm{s}^{-1}$ AGN. Upcoming Herschel observations will provide results for larger samples of such objects, where we will be able to investigate the relation above these AGN luminosities, i.e. on the "merger path". We might expect an upturn in host far-infrared luminosity which is suggested at $z \approx 1$ for more luminous AGN (Lutz et 2010), or the possible relation of host star formation to AGN obscuration (e.g. Page et al. 2001).

Acknowledgements. We thank the referee for helpful comments. PACS has been developed by a consortium of institutes led by MPE (Germany) and including UVIE (Austria); KUL, CSL, IMEC (Belgium); CEA, OAMP (France); MPIA (Germany); IFSI, OAP/OAT, OAA/CAISMI, LENS, SISSA (Italy); IAC (Spain). This development has been supported by the funding agencies BMVIT (Austria), ESA-PRODEX (Belgium), CEA/CNES (France), DLR (Germany), ASI (Italy), and CICYT/MCYT (Spain).

\section{References}

Alexander, D. M., Bauer, F. E., Brandt, W. N., et al. 2003, AJ, 125, 383 Barger, A. J., Cowie, L. L., Capak, P., et al. 2003, ApJ, 126, 632 Barger, A. J., Cowie, L. L., \& Wang, W.-H. 2008, ApJ, 689, 687 Bauer, F. E., Alexander, D. M., Brandt, W. N., et al. 2004, AJ, 128, 2048 Berta, S., et al. 2010, A\&A, 518, L30

Béthermin, M., Dole, H., Beelen, A., \& Aussel, H. 2010, A\&A, 512, A78

Bouché, N., Dekel, A., Genzel, R., et al. 2010, ApJ, accepted [arXiv: 0912.1858]

Cusumano, G. 2009, AIP Conf. Proc., 1126, 104

Hopkins, P. F., Hernquist, L., Cox, T. J., et al. 2006, ApJS, 163,1 Lutz, D., Sturm, E., Tacconi, L. J., et al. 2008, ApJ, 684, 853 Lutz, D., Mainieri, V., Rafferty, D., et al. 2010, ApJ, 712, 1287 Maiolino, R., Shemmer, O., Imanishi, M., et al. 2007, A\&A, 468, 979 Mullaney, J. R., Alexander, D. M., Huynh, M., Goulding, A. D., \& Frayer, D. 2010, MNRAS, 401, 995

Netzer, H. 2009, MNRAS, 399, 1907

Netzer, H., Lutz, D., Schweitzer, M., et al. 2007, ApJ, 666, 806

Page, M. J., Stevens, J. A., Mittaz, J. P. D., \& Carrera, F. J. 2001, Science, 294, 2516

Papovich, C., Rudnick, G., Le Floc'h, E., et al. 2007, ApJ, 668, 45

Pilbratt, G. L., et al. 2010, A\&A, 518, L1

Poglitsch, A., et al. 2010, A\&A, 518, L2

Rowan-Robinson, M. 1995, MNRAS, 272, 737

Sanders, D. B., Soifer, B. T., Elias, J. H., et al. 1988, ApJ, 325, 74

Serjeant, S., \& Hatziminaoglou, E. 2009, MNRAS, 397, 265

Wang, R., Carilli, C. L., Wagg, J., et al. 2008, ApJ, 687, 848 
1 MPE, Postfach 1312, 85741 Garching, Germany e-mail: shao@mpe.mpg.de

2 INAF - Osservatorio Astronomico di Roma, via di Frascati 33, 00040 Monte Porzio Catone, Italy

${ }^{3}$ Department of Physics, Durham University, South Road, Durham, DH1 3LE, UK

${ }^{4}$ European Space Astronomy Centre, Villafranca del Castillo, Spain

5 European Southern Observatory, Karl-Schwarzschild-Straße 2, 85748 Garching, Germany

6 INAF - Osservatorio Astronomico di Trieste, via Tiepolo 11, 34143 Trieste, Italy

7 IRFU/Service d'Astrophysique, Bât.709, CEA-Saclay, 91191 Gif-sur-Yvette Cedex, France
8 Pontificia Universidad Católica de Chile, Departamento de Astronomía y Astrofísica, Casilla 306, Santiago 22, Chile

9 Instituto de Astrofísica de Canarias, 38205 La Laguna, Spain

10 Departamento de Astrofísica, Universidad de La Laguna, Spain

11 Department of Astronomy and Astrophysics, 525 Davey Lab, Pennsylvania State University, University Park, PA 16802, USA

12 Istituto Nazionale di Astronomia, Osservatorio Astronomico di Bologna, via Ranzani 1, 40127 Bologna, Italy

13 Dipartimento di Astronomia, Universitá di Padova, 35122 Padova, Italy

14 Max-Planck-Institut für Plasmaphysik, Boltzmannstraße 2, 85748 Garching, Germany 